\title{
A Particular Matrix, Its Inversion and Some Norms
}

\author{
Seyyed Hossein Jafari-Petroudi ${ }^{1}$, Behzad Pirouz ${ }^{2}$ \\ ${ }^{1}$ Department of Mathematics, Payame Noor University, P. O. Box, 1935-3697, Tehran, Iran \\ ${ }^{2}$ Department of Mathematics, Azad University of Karaj, Karaj, Iran
}

Email address:

Hossein_5798@yahoo.com (S. H. J. Petroudi), behzadpirouz@gmail.com (B. Pirouz)

\section{To cite this article:}

Seyyed Hossein Jafari-Petroudi, Behzad Pirouz. A Particular Matrix, Its Inversion and Some Norms. Applied and Computational Mathematics. Vol. 4, No. 2, 2015, pp. 47-52. doi: 10.11648/j.acm.20150402.13

\begin{abstract}
In this note we study a new $\mathrm{n} \times \mathrm{n}$ matrix of the form $A=\left[\mathrm{a}^{\min (\mathrm{i}, \mathrm{j})-1}\right]_{\mathrm{i}, \mathrm{j}=1}^{\mathrm{n}}$, where a>1 is a real positive constant. We find determinant and inversion of this matrix and its Hadamard inverse. Then some bounds for the spectral norm of this matrix are presented. Finally we represent some properties of particular block diagonal matrices that their diagonal elements are these matrices.
\end{abstract}

Keywords: Positive Definite Matrix, Spectral Norm, Hadamard Inverse, Determinant, Block Diagonal

\section{Introduction}

Akbulak and Bozkort [1] studied Toeplitz matrices involving Fibonacci and Lucas numbers. They have found upper and lower bounds for the spectral norm of these matrices. Akbulak [2] studied Hadamard exponentioal matrix of the form $e^{\circ H_{n}}=\left[e^{i+j}\right]_{i, j}$ and found $l_{p}$ norm, two upper bounds for spectral norm and eigenvalues of this matrix. Bozkurt in [3] investigated $l_{p}$ norms of almost CauchyToeplitz matrices. Solak and Bozkort [4] determined bounds for the spectral and $l_{p}$ norms of Cauchy-Hankel matrices of the form $H_{n}=\left[\frac{1}{g+k h}\right]_{i, j=0}^{n}$, where $\mathrm{k}$ is defined by $i+j=k$ and $g, h$ are any positive numbers. Civciv and Turkmen [6] established a lower bound and upper bound for the $l_{p}$ norms of the Khatri-Rao product of Cauchy-Hankel matrix of the form $H_{n}=\left[\frac{1}{0 / 5+i+j}\right]_{i, j=0}^{n}$. Solak and Bahsi [9] studied the matrix of the form $B=\left[b_{i j}\right]$ where $b_{i j}=a+\min (i, j)-1$. They obtained its Euclidean norm and inversion of this matrix.

For more information one can see $[1-5,7,8,10]$.

In this paper we define a particular $n \times n$ matrix $A=\left[a_{i j}\right]=\left[a^{\min (i, j)-1_{i j}}\right]_{i, j=1}^{n}$, where $a>1$ is a real positive number, in exact of the form

$$
A=\left[\begin{array}{cccc}
1 & 1 & 1 \cdots & 1 \\
1 & a^{1} & a^{1} \ldots & a^{1} \\
1 & a^{1} & a^{2} \ldots & a^{2} \\
\vdots & \vdots & \vdots & \vdots \\
1 & a^{1} & a^{2} \cdots & a^{n-1}
\end{array}\right] .
$$

First by similar methods in some references which we illustrated in the beginning of this section we presented determinant of this matrix. Subsequently we show that this matrix is invertible and positive definite. Then we find some upper bounds and lower bounds for the spectral norm of this matrix. After that we get some properties of its Hadamard inverse, its product and the inversion of this matrix. Finally we study determinant and inversion of some block diagonal matrices that their diagonal elements are these matrices. All definitions and statements of this paper are available in references[1-4,7-11].

\section{Preliminary Definitions}

Let $A=\left[a_{i j}\right]$ is an $n \times n$ matrix, then the maximum column length norm $C_{1}$ (.) and maximum row length norm $r_{1}($.$) of$ $A$ is defined by

$$
C_{1}(A)=\max _{j} \sqrt{\sum_{i}\left|a_{i j}\right|^{2}}, r_{1}(A)=\max _{i} \sqrt{\sum_{j}\left|a_{i j}\right|^{2}} .
$$

Hadamard exponential and Hadamard inverse of this 
matrix are defined respectively by $e^{\circ A}=e^{a_{i j}}$ and $A^{\circ(-1)}=\left(\frac{1}{a_{i j}}\right)$. The $l_{p}$ norm of $A$ is defined by

$$
\|A\|_{p}=\left(\sum_{i=1}^{n} \sum_{j=1}^{n}\left|a_{i j}\right|^{p}\right)^{\frac{1}{p}} .
$$

For $p=2$ this norm is called Frobenius or Euclidean norm and showed by $\|A\|_{F}$. The spectral norm of $A$ is defined by

$$
\|A\|_{2}=\sqrt{\max _{1 \leq i \leq n}\left|\lambda_{i}\right|}
$$

Where $\lambda_{i}$ are the eigenvalues of matrix $A A^{H}$. Also $A^{H}$ is conjugate transpose of $A$. The inequality between the Frobenius and spectral norm is

$$
\frac{1}{\sqrt{n}}\|A\|_{F} \leq\|A\|_{2} \leq\|A\|_{F}
$$

Let $A=\left[a_{i j}\right]$ and $B=\left[b_{i j}\right]$ are $m \times n$ matrices. Hadamard product of $A$ and $B$ is defined by $A \circ B=\left[a_{i j} b_{i j}\right]$. Let $A, B$ and $C$ are $m \times n$ matrices and $A=B \circ C$. Then we have

$$
\|A\|_{2} \leq r_{1}(B) \cdot c_{1}(C) \text {. }
$$

It is known that

$$
\begin{aligned}
& \sum_{k=1}^{n-1} x^{k}=x+x^{2}+\cdots+x^{n-1}=\frac{x^{n}-x}{x-1}, \\
& \sum_{k=1}^{n-1} k x^{k}=\frac{(n-1) x^{n+1}-n x^{n-1}+x}{(x-1)^{2}} .
\end{aligned}
$$

\section{Main Results}

Theorem(3.1). Let $A$ be as in (1), then

$$
\operatorname{det}(A)=(a-1)^{n-1} \cdot a^{\frac{(n-1)(n-2)}{2}} .
$$

Proof. By definition of $A$ we have

$$
\operatorname{det}(A)=\operatorname{det}\left[\begin{array}{cccc}
1 & 1 & 1 \cdots & 1 \\
1 & a^{1} & a^{1} \cdots & a^{1} \\
1 & a^{1} & a^{2} \ldots & a^{2} \\
\vdots & \vdots & \vdots & \vdots \\
1 & a^{1} & a^{2} \cdots & a^{n-1}
\end{array}\right]
$$

By using elementary row operations we have

$$
\operatorname{det}(A)=\operatorname{det}\left[\begin{array}{ccccc}
1 & 1 & 1 & \cdots & 1 \\
a-1 & 0 & 0 & \cdots & 0 \\
a^{2}-1 & a^{2}-a & 0 & \cdots & 0 \\
\vdots & \vdots & \vdots & \vdots & \vdots \\
a^{n-1}-1 & a^{n-1}-a & a^{n-1}-a^{2} & \cdots & 0
\end{array}\right] .
$$

By expanding determinant of this matrix we obtain

$$
\operatorname{det}(A)=(a-1)^{n-1} \cdot a^{\frac{(n-1)(n-2)}{2}} .
$$

Corollary (3.2). Let $\Delta_{i}$ denotes the $i$ th leading principal minors of $A$, in particular, $\Delta_{1}=1, \ldots, \Delta_{n}=\operatorname{det}(A)$. Then we have

$$
\begin{aligned}
& \text { 1) } \Delta_{n} \Delta_{n-2} \geq \Delta_{n-1}^{2}, \\
& \text { 2) } \Delta_{n}^{\frac{1}{n}}>\Delta_{n-1}^{\frac{1}{n-1}}>\Delta_{n-2}^{\frac{1}{n-2}}>\cdots>\Delta, \\
& \text { (3) } \Delta_{n} \Delta_{n-2}=a \Delta_{n-1}^{2} .
\end{aligned}
$$

Proof. By definition of principal minors and theorem (3.1) we have

(1)

$$
\begin{aligned}
& \Delta_{n} \Delta_{n-2}=(a-1)^{n-1} \cdot a^{\frac{(n-1)(n-2)}{2}}(a-1)^{n-3} \cdot a^{\frac{(n-3)(n-4)}{2}} \\
& =(a-1)^{2(n-2)} \cdot a^{n^{2}-5 n+7} \geq(a-1)^{2(n-2)} \cdot a^{n^{2}-5 n+6} \\
& =(a-1)^{2(n-2)} \cdot a^{\frac{2(n-2)(n-3)}{2}}=\Delta_{n-1}^{2} .
\end{aligned}
$$

Thus the proof of (1) is completed.

Proof of (3) is clear by definition of $\Delta_{n}$. To proving (2) we just prove $\Delta_{n}^{\frac{1}{n}} \geq \Delta_{n-1}^{\frac{1}{n-1}}$. The rest of proof is similar to this part.

For positive integer $n>1$ we have

$$
\begin{aligned}
& (n-1)(n-1)=n^{2}-2 n+1>n^{2}-2 n \\
& =n(n-2) \\
& (n-1)(n-2)(2 n-2)=2 n^{3}-8 n^{2}+10 n-4 \\
& \geq 2 n^{3}-10 n^{2}+12 n=2 n(n-2)(n-3) .
\end{aligned}
$$

So we have

$$
\frac{n-1}{n}>\frac{n-2}{n-1}, \frac{(n-1)(n-2)}{2 n} \geq \frac{(n-2)(n-3)}{2 n-2} .
$$

Thus we get

$$
\Delta_{n}^{\frac{1}{n}}=(a-1)^{\frac{n-1}{n}} \cdot a^{\frac{(n-1)(n-2)}{2 n}}>(a-1)^{\frac{n-2}{n-1}} a^{\frac{(n-2)(n-3)}{2(n-1)}}=\Delta_{n-1}^{\frac{1}{n-1}} .
$$

Theorem(3.3). Let $A$ be as in (1), then $A$ is invertible and the inversion of $A$ is a tridiagonal matrix of the form 


$$
\begin{aligned}
& B=A^{-1} \\
& \left\{\begin{array}{ll}
b_{11}=\frac{a}{a-1} & \text { for }|i-j|>1 \\
b_{i j}=0 & \text { for } \quad 1<i=j<n \\
b_{i j}=\frac{(a+1)}{a^{i}(a-1)} & \text { for } \quad|i-j|=1 \\
b_{i j}=\frac{-1}{a^{i-1}(a-1)} & \\
b_{n n}=\frac{1}{a^{n-1}(a-1)} &
\end{array} .\right.
\end{aligned}
$$

Proof. By theorem (3.1) it is clear that $A$ is invertible. First we need a lemma from matrix algebra to prove the theorem.

Lemma(3.4). Let $A$ is an $n \times n$ nonsingular matrix, $b$ is an $n \times 1$ matrix and $c$ is a real number. If we take $M=\left[\begin{array}{ll}A & b \\ b^{T} & c\end{array}\right]$ then we have

$$
M^{-1}=\left[\begin{array}{cc}
A^{-1}+\frac{1}{k} A^{-1} b b^{T} A^{-1} & -\frac{1}{k} A^{-1} b \\
-\frac{1}{k} b^{T} A^{-1} & \frac{1}{k}
\end{array}\right],
$$

where $k=c-b^{T} A^{-1} b$.

Proof. By multiplying of two matrices $M, M^{-1}$ we have

$$
\left[\begin{array}{ll}
A & b \\
b^{T} & c
\end{array}\right] \cdot\left[\begin{array}{cc}
A^{-1}+\frac{1}{k} A^{-1} b b^{T} A^{-1} & -\frac{1}{k} A^{-1} b \\
-\frac{1}{k} b^{T} A^{-1} & \frac{1}{k}
\end{array}\right]=I_{n+1} .
$$

Thus the proof is completed.

Now by principal of mathematical induction on $n$ we prove theorem (3.2).

(Proof of theorem (3.2)). The result is true for $n=2$ that is

$$
A=\left[\begin{array}{ll}
1 & 1 \\
1 & a^{1}
\end{array}\right] \text { and } A^{-1}=\left[\begin{array}{cc}
\frac{\mathrm{a}}{\mathrm{a}-1} & \frac{-1}{\mathrm{a}-1} \\
\frac{-1}{\mathrm{a}-1} & \frac{1}{\mathrm{a}-1}
\end{array}\right] .
$$

Now assume that the result is true for $n-1$, that is $A=\left[a^{\min (i, j)-1}\right]_{i, j=0}^{n-1}, A^{-1}=\left(\left[a^{\min (i, j)-1}\right]_{i, j=0}^{n-1}\right)^{-1}$. Now by taking

$b^{T}=\left[\begin{array}{llll}a^{0} & a^{1} & \cdots & a^{n-1}\end{array}\right], c=a^{n}, A^{-1} b=\left[\begin{array}{llll}0 & 0 & \cdots & 1\end{array}\right]^{T}$,

Also by determining

$$
\begin{aligned}
& b^{T} A^{-1}=\left[\begin{array}{llll}
0 & 0 & \cdots & 0
\end{array}\right], k=a^{n}-b^{T} A^{-1} b \\
& =a^{n}-\left[\begin{array}{lllll}
0 & 0 & \cdots & 0 & 1
\end{array}\right]\left[a^{0}, a^{1}, \ldots, a^{n-1}\right]^{T} \\
& =a^{n}-a^{n-1}=a^{n-1}(a-1) \text {. }
\end{aligned}
$$

We get

$$
\frac{1}{k}=\frac{1}{a^{n-1}(a-1)}
$$

By substituting these values along with lemma (3.4) we get the result.

Theorem(3.5). Let $A$ be as in (1), then the $l_{p}$ norm of $A$ is

$$
\|A\|_{p}=\left[\frac{a^{(n+1) p}+a^{n p}-(2 n+1) a^{p}+2 n-1}{\left(a^{p}-1\right)^{2}}\right]^{\frac{1}{p}} .
$$

Proof. By definition of $A$ and $l_{p}$ norm we have

$$
\begin{aligned}
\|A\|_{p}^{p} & =\left((2 n-1)+(2 n-3) a^{p}+(2 n-5) a^{2 p}+\right. \\
\ldots+ & \left.3 a^{(n-2) p}+a^{(n-1) p}\right)=\sum_{k=1}^{n}(2 k-1) a^{(n-k) p} . \\
& =\left(a^{n p}\left(2 \sum_{k=1}^{n} k\left(a^{-p}\right)^{k}-\sum_{k=1}^{n}\left(a^{-p}\right)^{k}\right) .\right.
\end{aligned}
$$

According to equalities (7) we obtain

$$
\begin{aligned}
& \|A\|_{p}^{p}= \\
& {\left[a ^ { n p } \left[2 \frac{(n-1) a^{-p(n+1)}-n a^{-n p}+a^{-p}}{\left(a^{-p}-1\right)^{2}}+2 n a^{-n p}\right.\right.} \\
& \left.-\frac{\left.\left.a^{-(n+1) p}-a^{-p}\right]\right]}{a^{-p}-1}\right] \\
& =\left[a^{n p} \frac{(2 n-1) a^{-(n+2) p}-(2 n+1) a^{-(n+1) p}+a^{-2 p}+a^{-p}}{\left(a^{-p}-1\right)^{2}}\right] \\
& =\left[\frac{a^{(n-1) p}+a^{(n-2) p}+(2 n-1) a^{-2 p}-(2 n+1) a^{-p}}{\left(a^{-p}-1\right)^{2}}\right] .
\end{aligned}
$$

By taking $\frac{1}{p}$ th power from the both sides of above equality we have

$$
\left\|a^{\circ H_{n}}\right\|_{p}=\left[\frac{a^{(n-1) p}+a^{(n-2) p}+(2 n-1) a^{-2 p}-(2 n+1) a^{-p}}{\left(a^{-p}-1\right)^{2}}\right]^{\frac{1}{p}} .
$$

If we rephrase the last equality we obtain

$$
\|A\|_{p}=\left[\frac{a^{(n+1) p}+a^{n p}-(2 n+1) a^{p}+2 n-1}{\left(a^{p}-1\right)^{2}}\right]^{\frac{1}{p}} .
$$

Corollary(3.6). The Euclidean norm of $A$ is 


$$
\|A\|_{F}=\left[\frac{a^{2(n+1)}+a^{2 n}-(2 n+1) a^{2}+2 n-1}{\left(a^{2}-1\right)^{2}}\right]^{\frac{1}{2}} .
$$

Proof. If we set $p=2$ in (8) then we get the Euclidean norm of $A$.

Theorem (3.7). Let $A$ be as in (1), then we have

$$
\begin{aligned}
& \frac{1}{\sqrt{n}}\left[\frac{a^{2(n+1)}+a^{2 n}-(2 n+1) a^{2}+2 n-1}{\left(a^{2}-1\right)^{2}}\right]^{\frac{1}{2}} \leq\|A\|_{2} \\
& \leq\left[\frac{a^{2(n+1)}+a^{2 n}-(2 n+1) a^{2}+2 n-1}{\left(a^{2}-1\right)^{2}}\right]^{\frac{1}{2}} .
\end{aligned}
$$

Proof. It follows from corollary (3.6) and (5).

Theorem(3.8). Let $A$ be as in (1) then we have the following upper bound for spectral norm of $A$.

$$
\|A\|_{2} \leq \sqrt{\frac{a^{2 n}-1}{a^{2}-1}} \sqrt{\left(\frac{a^{2 n-2}-1}{a^{2}-1}\right)+1} .
$$

Proof. By definition of $A$ we have

$$
\begin{aligned}
& A=\left[\begin{array}{cccc}
a^{0} & a^{0} & \cdots & a^{0} \\
a^{0} & a^{1} & \cdots & a^{1} \\
a^{0} & a^{1} & \cdots & a^{2} \\
\vdots & \vdots & \vdots & \vdots \\
a^{0} & a^{1} & \cdots & a^{n-1}
\end{array}\right] \\
& =\left[\begin{array}{cccc}
a^{0} & a^{0} & \cdots & 1 \\
a^{0} & a^{1} & \cdots & 1 \\
a^{0} & a^{1} & \cdots & 1 \\
\vdots & \vdots & \vdots & \vdots \\
a^{0} & a^{1} & \cdots & a^{n-1}
\end{array}\right] \circ\left[\begin{array}{cccc}
1 & 1 & \cdots & a^{0} \\
1 & 1 & \cdots & a^{1} \\
1 & 1 & \cdots & a^{2} \\
\vdots & \vdots & \vdots & \vdots \\
1 & 1 & \cdots & 1
\end{array}\right] \\
& =C \circ B
\end{aligned}
$$

By definition of row maximum length norm and column maximum length norm we have

$$
\begin{aligned}
& r_{1}(C)=\max _{i} \sqrt{\sum_{j}\left|c_{i j}\right|^{2}}=\sqrt{\frac{a^{2 n}-1}{a^{2}-1}}, \\
& C_{1}(B)=\max _{j} \sqrt{\sum_{i}\left|b_{i j}\right|^{2}}=\sqrt{\left(\frac{a^{2 n-2}-1}{a^{2}-1}\right)+1} .
\end{aligned}
$$

According to (6) we know $\|A\|_{2} \leq r_{1}(C) c_{1}(B)$.

Thus we have

$$
\|A\|_{2} \leq \sqrt{\frac{a^{2 n}-1}{a^{2}-1}} \sqrt{\left(\frac{a^{2 n-2}-1}{a^{2}-1}\right)+1} .
$$

Corollary (3.9). Let $A$ be as in (1), then $A$ is positive definite matrix.

Proof. According to theorem (3.1), all leading principal

\begin{tabular}{|c|c|c|}
\hline $\mathrm{n}$ & $\operatorname{det}(\mathrm{A})$ & $\begin{array}{l}\text { Eigenvalues of each A (is rounded off to four } \\
\text { decimal places) }\end{array}$ \\
\hline 2 & 1 & $0.3820,2.6180$ \\
\hline 3 & 2 & $0.3542,1.000,5.6458$ \\
\hline 4 & 8 & $0.3532,0.8804,2.2275,11.5386$ \\
\hline 5 & 64 & $0.3535,0.8754,1.9227,4.6332,23.2152$ \\
\hline 6 & 1024 & $0.3535,0.8753,1.9069,3.9765,9.3839,46.5038$ \\
\hline 7 & 32768 & $\begin{array}{l}0.3535,0.8753,1.9067,3.9397,8.0429,18.8364 \text {, } \\
93.0454\end{array}$ \\
\hline 8 & 2097152 & $\begin{array}{l}0.3535,0.8753,1.9067,3.9393,7.9656,16.1396 \\
37.7100,186.1101\end{array}$ \\
\hline 9 & 268435456 & $\begin{array}{l}0.3535,0.8753,1.9067,3.9393,7.9646,15.9828, \\
32.3085,75.4393,372.2300\end{array}$ \\
\hline 10 & 68719476736 & $\begin{array}{l}0.3535,0.8753,1.9067,3.9393,7.9646,15.9808, \\
31.9939,64.6335,150.8886,744.4648\end{array}$ \\
\hline
\end{tabular}
minors of $A$ are positive, thus the result follows from [11].

Corollary (3.10). Let $A$ be as in (1), then all eigenvalues of $A$ are positive.

Proof. Since $A$ is positive definite, so the result follows from [11].

Example(3.11). Let $A$ be as in (1). Determinants and eigenvalues of $A$ for $a=2$ and some values of $n$ are represented in table1.

Table 1. Determinants and eigenvalues of $A$

Therem (3.11) Let $A$ be as in (1) and $\lambda_{n}$ be the largest eigenvalues of $A$, then we have

$$
\frac{a^{n}-2 a^{n-1}+1}{a-1} \leq \lambda_{n} \leq \frac{a^{n}-1}{a-1} .
$$

Proof. If we apply the well known Gershgorin's theorem for the last row of $A$, then we get

$$
\left|\lambda_{n}-a^{n-1}\right| \leq 1+a+a^{2}+\cdots+a^{n-2}=\frac{a^{n-1}-1}{a-1} .
$$

So we have

$$
a^{n-1}-\frac{a^{n-1}-1}{a-1} \leq \lambda_{n} \leq a^{n-1}+\frac{a^{n-1}-1}{a-1} .
$$

Thus we obtain

$$
\frac{a^{n}-2 a^{n-1}+1}{a-1} \leq \lambda_{n} \leq \frac{a^{n}-1}{a-1} .
$$

Hence we have an upper bound and a lower bound for the spectral radius of $A$.

Corollary (13). Let $A$ be as in (1) and $\rho(A)$ be the spectral radius of $A$, then we have

$$
\frac{a^{n}-2 a^{n-1}+1}{a-1} \leq \rho(A) \leq \frac{a^{n}-1}{a-1} .
$$


Proof. Since $\rho(A)=\max \left\{\left|\lambda_{i}\right| ; \lambda_{i}\right.$ is an eigenvalue of $A$, the for $i \in\{1,2, \ldots, n\}\}$,

result follows from theorem(3.11).

Theorem(3.14). Let $A$ be as in (1), then determinant of Hadamard inverse of $A$ is

$$
\operatorname{det}\left((A)^{\circ(-1)}\right)=(1-a)^{n-1} a^{-\frac{n(n-1)}{2}} .
$$

Proof. By definition of $(A)^{\circ(-1)}$ we have

$$
\left.\operatorname{det}\left(A^{\circ(-1)}\right)=\mid \begin{array}{cccc}
1 & 1 & 1 \cdots & 1 \\
1 & a^{-1} & a^{-1} \ldots & a^{-1} \\
1 & a^{-1} & a^{-2} \cdots & a^{-2} \\
\vdots & \vdots & \vdots & \vdots \\
1 & a^{-1} & a^{-2} \cdots & a^{-(n-1)}
\end{array}\right]
$$

By using elementary row operations we get

$$
\operatorname{det}\left(A^{\circ(-1)}\right)=\left\lfloor\left[\begin{array}{cccc}
1 & 1 & \cdots & 1 \\
a^{-1}-1 & 0 & \cdots & 0 \\
a^{-2}-1 & a^{-2}-a^{-1} & \ldots & 0 \\
\vdots & \vdots & \vdots & \vdots \\
a^{-(n-1)}-1 & a^{-(n-1)}-a^{-1} & \cdots & 0
\end{array}\right] .\right.
$$

Thus by expanding this determinant we have

$$
\operatorname{det}(A)^{\circ(-1)}=(1-a)^{n-1} a^{-\frac{n(n-1)}{2}} .
$$

So $A^{\circ(-1)}$ is nonsingular and $\left(A^{\circ(-1)}\right)^{-1}$ exists. So we have the following theorem.

Theorem(3.15). Let $A$ be as in (1). Then $A^{\circ(-1)}$ is invertible and the inversion of $A^{\circ(-1)}$ is a tridiagonal matrix of the form

$$
\begin{aligned}
& B=\left(A^{\circ(-1)}\right)^{-1} \\
& \begin{cases}b_{11}=\frac{-1}{a-1} & \text { for }|i-j|>1 \\
b_{i j}=0 & \text { for } 1<i=j<n \\
b_{i j}=\frac{-a^{i-1}(a+1)}{(a-1)} & \text { for }|i-j|=1 \\
b_{i j}=\frac{a^{i-1}}{(a-1)} & \\
b_{n n}=\frac{-a^{n-1}}{(a-1)} & \end{cases}
\end{aligned}
$$

Proof. The proof is similar to theorem (3.3).

Theorem(3.16). The $l_{p}$ norm of $A^{\circ(-1)}$ is

$$
\|\left(A^{\circ(-1)} \|_{p}=\left[\frac{a^{-(n+1) p}+a^{-n p}-(2 n+1) a^{-p}+2 n-1}{\left(a^{-p}-1\right)^{2}}\right]^{\frac{1}{p}} .\right.
$$

Proof. The proof is similar to theorem (3.5).

Theorem(3.17). Let $D$ is a block diagonal matrix of the form $D=\operatorname{diag}\left(A_{11}, A_{22}, \ldots, A_{m m}\right)$, where each $A_{i i}$, for $i=1,2, \ldots, m$ is defined as follows

$$
A_{i i}=\left[\begin{array}{cccc}
a^{(i-1) n} & a^{(i-1) n} & \cdots & a^{(i-1) n} \\
a^{(i-1) n} & a^{(i-1) n+1} & \cdots & a^{(i-1) n+1} \\
\vdots & \vdots & \vdots & \vdots \\
a^{(i-1) n} & a^{(i-1) n+1} & \cdots & a^{i n-1}
\end{array}\right] .
$$

Then we have

$$
\text { (1) } \begin{aligned}
\operatorname{det}(D)=\prod_{i=1}^{m} \operatorname{det} A_{i i} & =\left((a-1)^{n-1} a^{\frac{(n-1)(n-2)}{2}}\right)^{m} \prod_{i=1}^{m} a^{(i-1) n^{2}} \\
& =(a-1)^{m(n-1)} a^{\frac{m(n-1)(n-2)+m(m-1) n^{2}}{2}},
\end{aligned}
$$

(2) $D^{-1}=\operatorname{diag}\left(A_{11}^{-1}, A_{22}^{-1}, \ldots, A_{m m}^{-1}\right)$

$=\operatorname{diag}\left(A^{-1}, a^{-n} A^{-1}, a^{-2 n} A^{-1}, \ldots, a^{-(n-1) n} A^{-1}\right)$.

Proof. For block diagonal matrix $D=\operatorname{diag}\left(A_{11}, A_{22}, \ldots, A_{m m}\right)$, we have

(1) $\operatorname{det} D=\operatorname{det} A_{11} \cdot \operatorname{det} A_{22} \ldots \ldots \operatorname{det} A_{m m}$

$=\prod_{i=1}^{m} \operatorname{det} A_{i i}, \quad$ (2) $D^{-1}=\operatorname{diag}\left(A_{11}^{-1}, A_{22}^{-1}, \ldots, A_{m m}^{-1}\right)$. So we have

$$
\begin{aligned}
& \operatorname{det}(D)=\prod_{i=1}^{m} \operatorname{det}\left(A_{i i}\right) \\
& =\prod_{k=1}^{m} \operatorname{det}\left[\begin{array}{cccc}
a^{(i-1) n} & a^{(i-1) n} & \cdots & a^{(i-1) n} \\
a^{(i-1) n} & a^{(i-1) n+1} & \cdots & a^{(i-1) n+1} \\
\vdots & \vdots & \vdots & \vdots \\
a^{(i-1) n} & a^{(i-1) n+1} & \cdots & a^{i n-1}
\end{array}\right] \\
& =\prod_{k=1}^{m} \operatorname{det}\left(\left[\begin{array}{cccc}
1 & 1 & \cdots & 1 \\
1 & a^{1} & \cdots & a^{1} \\
1 & a^{1} & \cdots & a^{2} \\
\vdots & \vdots & \vdots & \vdots \\
1 & a^{1} & \cdots & a^{n-1}
\end{array}\right]\right) .
\end{aligned}
$$

From definition of $A$ as we see in (1) and its determinant from theorem (3.7) we have

$$
\begin{aligned}
& \operatorname{det}(D)=\prod_{i=1}^{m} a^{(i-1) n^{2}} \operatorname{det}\left(a^{\circ H_{n}}\right) \\
& =\left((a-1)^{n-1} a^{\frac{(n-1)(n-2)}{2}}\right)^{m} \prod_{i=1}^{m} a^{(i-1) n^{2}} \\
& =(a-1)^{m(n-1)} a^{\frac{m(n-1)(n-2)}{2}+\frac{(m-1)((m-1)+1) n^{2}}{2}} \\
& =(a-1)^{m(n-1)} a^{\frac{m(n-1)(n-2)+m(m-1) n^{2}}{2}} .
\end{aligned}
$$


Also for obtaining the inversion of each $A_{i i}$ we have

$$
\begin{aligned}
& A_{i i}^{-1}=\left[\begin{array}{cccc}
a^{(i-1) n} & a^{(i-1) n} & \cdots & a^{(i-1) n} \\
a^{(i-1) n} & a^{(i-1) n+1} & \cdots & a^{(i-1) n+1} \\
\vdots & \vdots & \vdots & \vdots \\
a^{(i-1) n} & a^{(i-1) n+1} & \cdots & a^{i n-1}
\end{array}\right]^{-1} \\
& =\left(a^{(i-1) n}\right)^{-1}\left[\begin{array}{cccc}
1 & 1 & \cdots & 1 \\
1 & a^{1} & \cdots & a^{1} \\
1 & a^{1} & \cdots & a^{2} \\
\vdots & \vdots & \vdots & \vdots \\
1 & a^{1} & \cdots & a^{n-1}
\end{array}\right]^{-1} \\
& =a^{-(i-1) n} A^{-1} .
\end{aligned}
$$

So we have

$$
\begin{aligned}
& D^{-1}=\operatorname{diag}\left(A_{11}^{-1}, A_{22}^{-1}, \ldots, A_{m m}^{-1}\right) \\
& =\operatorname{diag}\left(A^{-1}, a^{-n} A^{-1}, a^{-2 n} A^{-1}, \ldots, a^{-(n-1) n} A^{-1}\right)
\end{aligned}
$$

Thus the proof is completed.

Corrolary (3.18). Let $A$ is a block diagonal matrix of the form $D=\operatorname{diag}\left(A_{11}, A_{22}, \ldots, A_{m m}\right)$, where $A_{i i}$ be as in (1). Then we have

$$
\begin{aligned}
& \text { (1) } \operatorname{det}(D)=\prod_{i=1}^{m} \operatorname{det} A_{i i}=\left((a-1)^{n-1} a^{\frac{(n-1)(n-2)}{2}}\right)^{m} \\
& =(a-1)^{m(n-1)} a^{\frac{m(n-1)(n-2)}{2}}, \\
& \text { (2) } D^{-1}=\operatorname{diag}\left(A_{11}^{-1}, A_{22}^{-1}, \ldots, A_{m m}^{-1}\right) \\
& =\operatorname{diag}\left(A^{-1}, A^{-1}, \ldots, A^{-1}\right) .
\end{aligned}
$$

Proof. The result is concluded from theorem (3.17). Example(3.19). Let $D=\operatorname{diag}\left(A_{11}, A_{22}\right)$, in exact

$$
D=\left[\begin{array}{cccccc}
1 & 1 & 1 & 0 & 0 & 0 \\
1 & a^{1} & a^{1} & 0 & 0 & 0 \\
1 & a^{1} & a^{2} & 0 & 0 & 0 \\
0 & 0 & 0 & a^{3} & a^{3} & a^{3} \\
0 & 0 & 0 & a^{3} & a^{4} & a^{4} \\
0 & 0 & 0 & a^{3} & a^{4} & a^{5}
\end{array}\right] .
$$

According to theorem (3.17) we get

$$
\begin{gathered}
\operatorname{det}(D)=\operatorname{det} A_{11} \operatorname{det} A_{22} \\
=\left[a(a-1)^{2}\right] \cdot\left[a^{9} a(a-1)^{2}\right]=(a-1)^{4} a^{11}, \\
D^{-1}=\operatorname{diag}\left(A_{11}^{-1}, a^{-3} A_{11}^{-1}\right)
\end{gathered}
$$

$$
=\operatorname{diag}\left(\frac{1}{a-1}\left[\begin{array}{ccc}
a & -1 & 0 \\
-1 & \frac{a+1}{a} & \frac{-1}{a} \\
0 & \frac{-1}{a} & \frac{1}{a}
\end{array}\right], \frac{a^{-3}}{a-1}\left[\begin{array}{ccc}
a & -1 & 0 \\
-1 & \frac{a+1}{a} & \frac{-1}{a} \\
0 & \frac{-1}{a} & \frac{1}{a}
\end{array}\right]\right) \text {. }
$$

\section{Conclusion}

In this paper we investigated some properties of a new special matrix. We found remarkable relations about this matrix. We saw that this matix is positive definite and has a tridiagonal inversion. Also its inversion is a symmetric matrix. In addition, diagonal matrices that their entries are theses matrices have interesting property too.

\section{References}

[1] M. Akbulak, D. Bouzkurt, On the Norms of Toeplitz Matrices Involving Fibonacci and Lucas Numbers, HACET J MATH STAT, Vol 37,(2),(2008), 89-95

[2] M. Akbulak, A. Ipek, Hadamard Exponential Hankel Matrix, Its Eigenvalues and Some Norms, Math. Sci. Lett., Vol 1, No. $1,(2012), 81-87$

[3] D. Bozkurt, On the $l_{p}$ Norms of Almost Cauchy-Toeplitz Matrices, Tr. J. of Mathematics, 20, (1996), 545-552

[4] D. Bozkurt, S. Solak, A Note on Bound for Norms of CuachyHankel Matrices, Numerical Linear Algebra, vol. ED-10, (2003), 377-382

[5] D. Bozkurt, A Note on the Spectral Norms of the Matrices Connected Integer Numbers Sequence, Math.GM, vol. ED-1, University Science, (2011), 1-4

[6] H. Civciv, R. Turkmen, On the Bounds for the Spectral and $l_{p}$ Norms of the Khatri-Rao Products of Cauchy-Hankel Matrices, J.I.P.AM, 195, vol. ED-7, (2006), 1-11.

[7] A. Nalli, M. Sen, On the Norms of Circulant Matrices with Generalized Fibonacci Numbers, Selçuk J. Appl. Math, vol. 11, no. $1,(2010), 107-116$

[8] S. Solak, B. Mustafa, On the Spectral Norms of Toeplitz Matrices with Fibonacci and Lucas Number, HACET J MATH STAT, Vol 42, (1), (2013), 15-19

[9] S. Solak, B. Mustafa, A Particular Matrix and its Some Properties, Scientific Research and Essays, vol. 8, no. 1, (2013), 1-5

[10] S. Solak, R. Turkmen, D. Bozkurt, Upper Bounds for the Spectral and $l_{p}$ Norms of Cauchy-Toeplitz and CauchyHankel Matrices, Mathematical \& Computational Applications, Vol 9, No.1, (2004), 41-47

[11] F. Zhang, Matrix Theory Basic Results and Techniques, Springer Science+ Business Media, New York, (2011). 